\title{
Inflammatory pseudotumor in the submandibular region: A case report with immunohistochemical findings
}

\author{
Itaru Tojyo, Kenjiro Okamoto, Yuji Shinohara, Yukihiro Hiraishi, Norifumi Kiga, Shigeyuki Fujita \\ Department of Oral and Maxillofacial Surgery, Wakayama Medical University, Kimiidera, Wakayama, Japan
}

Received: May 25, 2016

DOI: $10.5430 /$ css.v2n3p18
Accepted: June 16, 2016

Online Published: June 22, 2016

URL: http://dx.doi.org/10.5430/css.v2n3p18

\begin{abstract}
Background: Inflammatory pseudotumor is a rare, benign non-neoplastic lesion. Clinically it behaves as an aggressive lesion, which can be mistaken for malignant tumor.

Case presentation: This article presents a rare case of submandibular inflammatory pseudotumor in a 44-year-old man patient. The submandibular mass presented rapid enlargement in one month's duration after initial examination. The patient was treated with surgery. Histologic examination of the resected specimen showed florid myofibroblastic and fibrohistiocytic proliferations in an inflammatory background. Immunohistochemically, the cells expressed vimentin, $\alpha$-smooth muscle actin and CD68 indicating a diagnosis of inflammatory pseudotumor. The patient had an uneventful postoperative recovery and no evidence of recurrence. Conclusions: We report a case of inflammatory pseudotumor occurring at the site of submandibular region.
\end{abstract}

Key Words: Inflammatory pseudotumor, Inflammatory myofibroblastic tumor, Submandibular tumor, Immunohistochemistry

\section{INTRODUCTION}

Inflammatory pseudotumor is nonneoplastic lesion that mimics malignant neoplastic processes due to the infiltrating and destructive nature. Inflammatory pseudotumor can be found anywhere in the body, although it is most commonly located in the head and neck region. Inflammatory pseudotumor etiology is unknown and terninology includes plasma cell granulomas, xanthogranuloma, fibrous xanthoma, and inflammatory myofibroblastic tumors.

We present a very rare case of a submandibular inflammatory pseudotumor in a 45-year-old man. The lesion presents a rapidly growing mass with aggressive behavior, which mimicked a malignant neoplasm.

\section{CASE REPORT}

A 44-year-old man was referred to another dentistry hospital because of swelling in the right submandibular region in November, 1996. He was administered antibiotics, he consulted our clinic in December, 1996. In our initial examination, the mass size was $15 \mathrm{~mm} \times 20 \mathrm{~mm}$ in diameter, which was elastic hard, and mobile, and was painful on palpation, but caused non-spontaneous pain. There was no obstruction of salivary flow and no salivary calculus. A first laboratory examination indicated a white blood cell (WBC) count of $5,400 / \mathrm{mm}^{3}$, and C-reactive protein (CRP) of $0.0 \mathrm{mg} / \mathrm{dl}$. One month later, the mass increased in size to $45 \mathrm{~mm} \times 60 \mathrm{~mm}$ in diameter (see Table 1). Based on these clinical findings, we suspected a specific disease or malignant tumor (see

${ }^{*}$ Correspondence: Itaru Tojyo; Email: kagoky@ wakayama-med.ac.jp; Address: Department of Oral and Maxillofacial Surgery, Wakayama Medical University, 811-1, Kimiidera, Wakayama 641-8509, Japan. 
Figure 1).

Table 1. Laboratory data and the mass size

\begin{tabular}{llll}
\hline Date & CRP & WBC & Size \\
\hline Initial examination & 0 & 5,400 & $15 \mathrm{~mm} \times 20 \mathrm{~mm}$ \\
26 days later & 0.1 & 8,100 & $30 \mathrm{~mm} \times 40 \mathrm{~mm}$ \\
36 days later & 0.2 & 5,900 & $45 \mathrm{~mm} \times 60 \mathrm{~mm}$ \\
\hline
\end{tabular}

Note. CRP: C-reactive protein; WBC: white blood cell

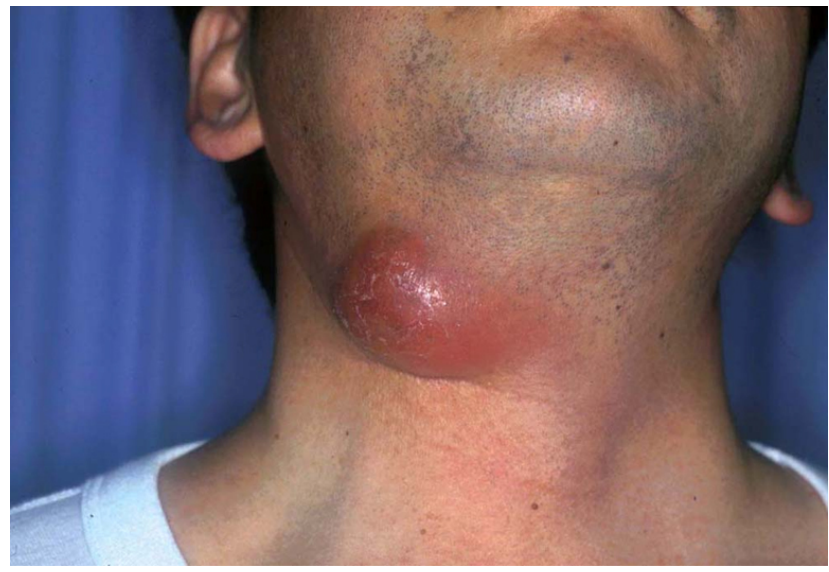

Figure 1. The right submandibular mass

A computed tomography (CT) scan documented an oval located in the inferior part of the submandibular gland. There was no abnormal lymph node swelling. The mass was suspected to be a right submandibular inflammatory tumor (see Figure 2).

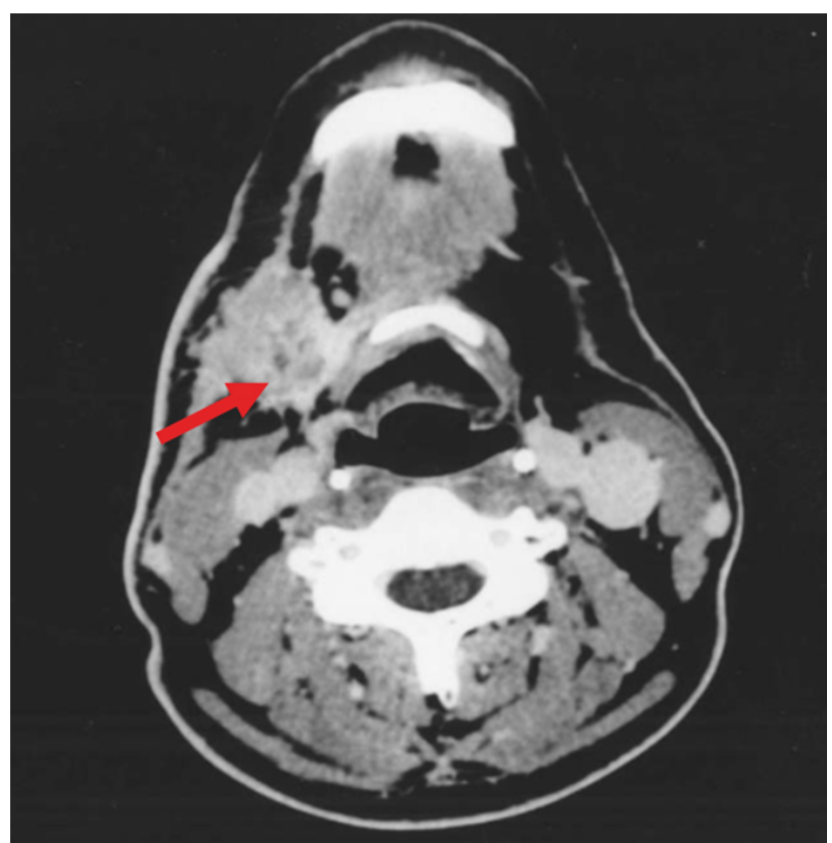

Figure 2. CT indicates an oval located in the inferior part of the submandibular gland (arrow)

Published by Sciedu Press

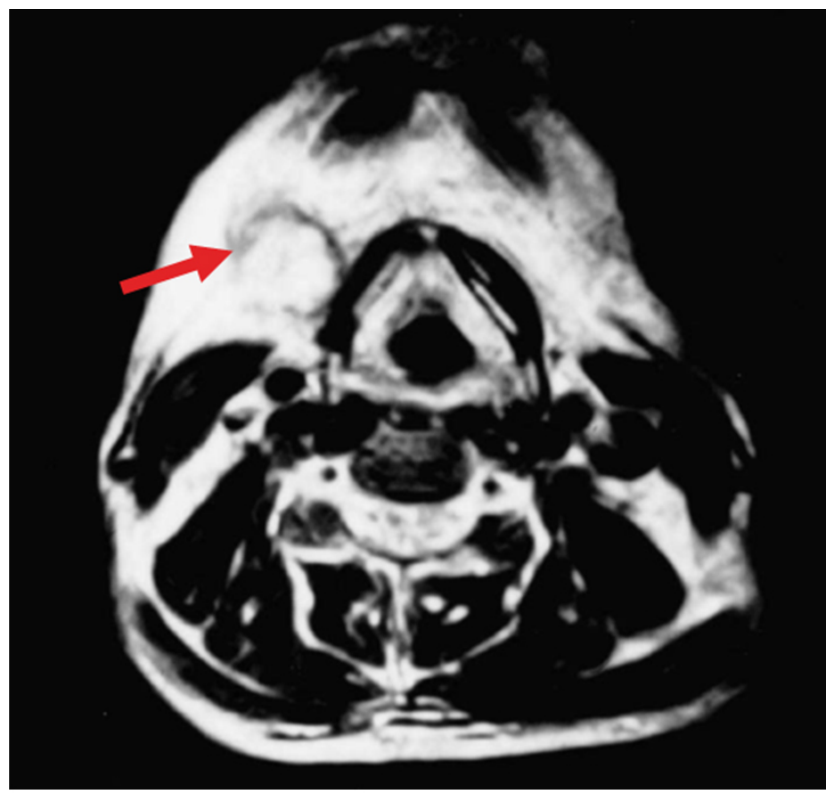

Figure 3. MRI indicates that the mass is low intensity as muscle on $\mathrm{T} 1$ and a high intensity as subcutaneous fat tissue on T2 (arrow)

Magnetic resonance imaging (MRI) demonstrated that the mass was low intensity as muscle on $\mathrm{T} 1$ and high intensity as subcutaneous fat tissue on T2. Probably the mass would probably have been diagnosed as an abscess rather than a tumor (see Figure 3).

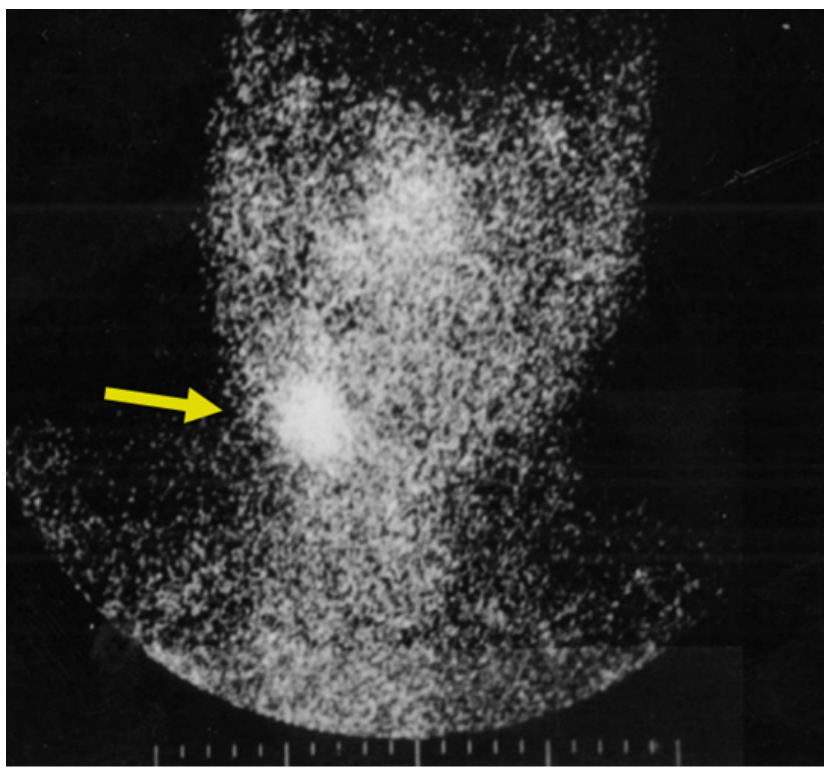

Figure 4. ${ }^{67} \mathrm{Ga}$ scintigraphy demonstrates that intensive uptake in the right submandibular region (arrow)

${ }^{67}$ Ga scintigraphy demonstrated intensive uptake in the same region (see Figure 4). 
$\mathrm{T}_{C} \mathrm{O}_{4}^{-}$scintigraphy showed slight uptake in the right submandibular region, probably the outside of submandibular gland. The uptake resembled that of a Wartin tumor (see Figure 5).

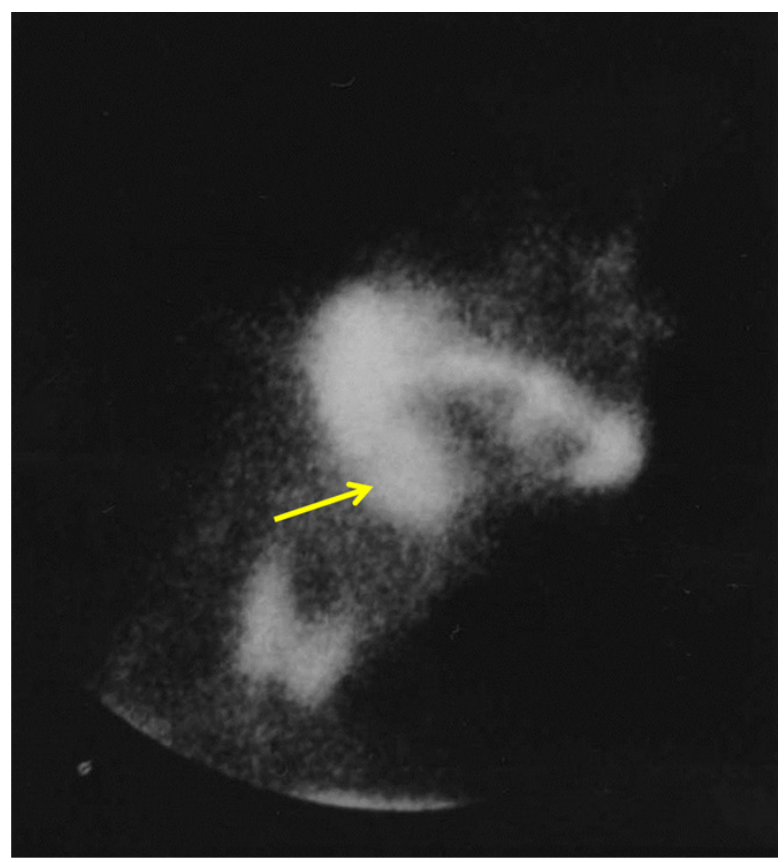

Figure 5. $\mathrm{T}_{C} \mathrm{O}_{4}^{-}$scintigraphy shows a slight uptake in the right submandibular region (arrow)

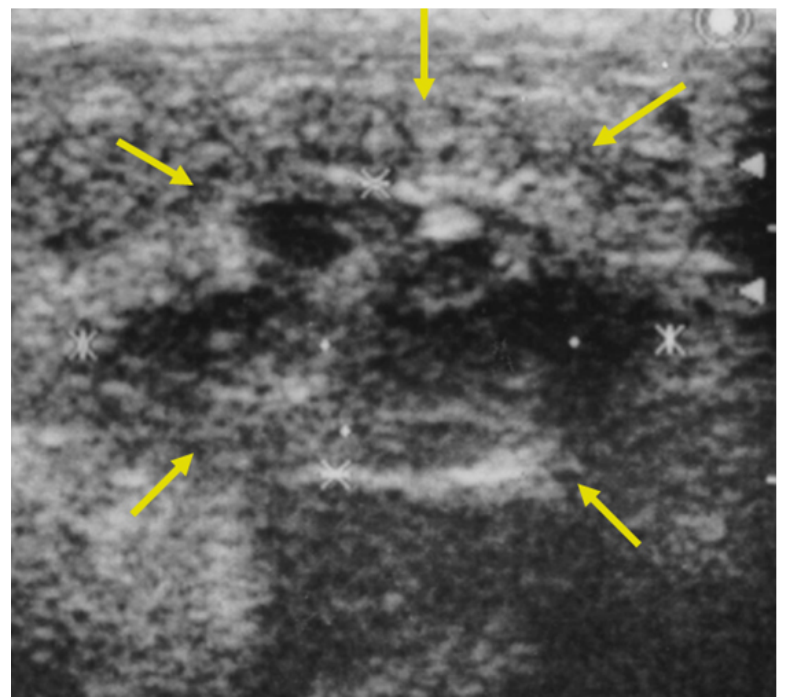

Figure 6. Ultrasonography indicated that the bottom echo is enhanced and the mass lesion is heterogeneous (arrows)

Ultrasonography indicated that the bottom echo was enhanced and the mass lesion was heterogeneous (see Figure 6).

On January 1997, tumor resection was performed under general anesthesia. The mass strongly adhered to the contiguous tissue, but it was excised with the right submandibular gland. The size of the resection was $8 \mathrm{~cm} \times 4 \mathrm{~cm}$ in diameter. After the operation, there was no complication, and no recurrence of the tumor has been noted for two years.

The pathohistological diagnosis of the removed lesion was an abscess: the histologic examination with hematoxylin and eosin staining showed that many neutrophils and plasma cells infiltrated the subcutaneous region (see Figure 7).

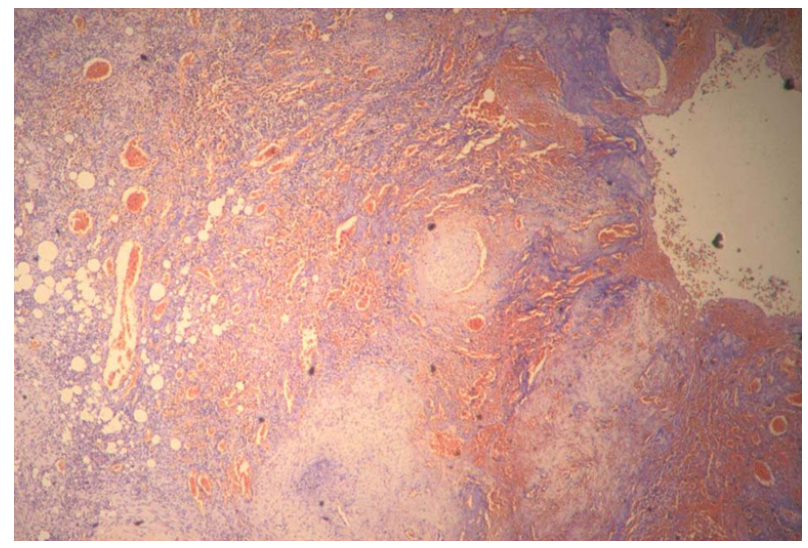

Figure 7. Many neutrophils and plasma cells infiltration (hematoxylin and eosin staining, $\times 20$ )

Immunohistochemical staining was undertaken for the following: vimentin (DAKO A/S Glostrup Denmark), strongly positive in fibroblast cells around the inflammation region (see Figure 8A); $\alpha$-smooth muscle actin (DAKO A/S Glostrup Denmark), strongly positive around bloodcorpuscle constituents (see Figure 8B); CD68 (PGM1) (DAKO A/S Glostrup Denmark), positive scattering at the inflammation region (see Figure 8C); CD68 (KP1) (DAKO A/S Glostrup Denmark), negative; proliferating cell nuclear antigen (PCNA) (DAKO A/S Glostrup Denmark), negative; and S-100 (DAKO A/S Glostrup Denmark), negative. The immunohistochemical staining color was developed by 3,3'-diaminobenzidine (DAB) $/ \mathrm{H}_{2} \mathrm{O}_{2}$ solution followed by counterstaining with methyl green.

In consideration of both clinical and pathohistological findings, the lesion was finally diagnosed as an inflammatory pseudotumor.

\section{DisCUSSION}

Inflammatory pseudotumors most commonly occur in the lung, but have also been encountered in a wide variety of sites. ${ }^{[1]}$ Synonyms for an inflammatory pseudotumor are plasma cell granuloma, xanthomatous pseudotumor, pseudosarcomatous myofibroblastic proliferation, inflammatory myofibroblastic proliferation, and recently, inflammatory myofibroblastic cell tumor and inflammatory fibrosarcoma. ${ }^{[2]}$ 

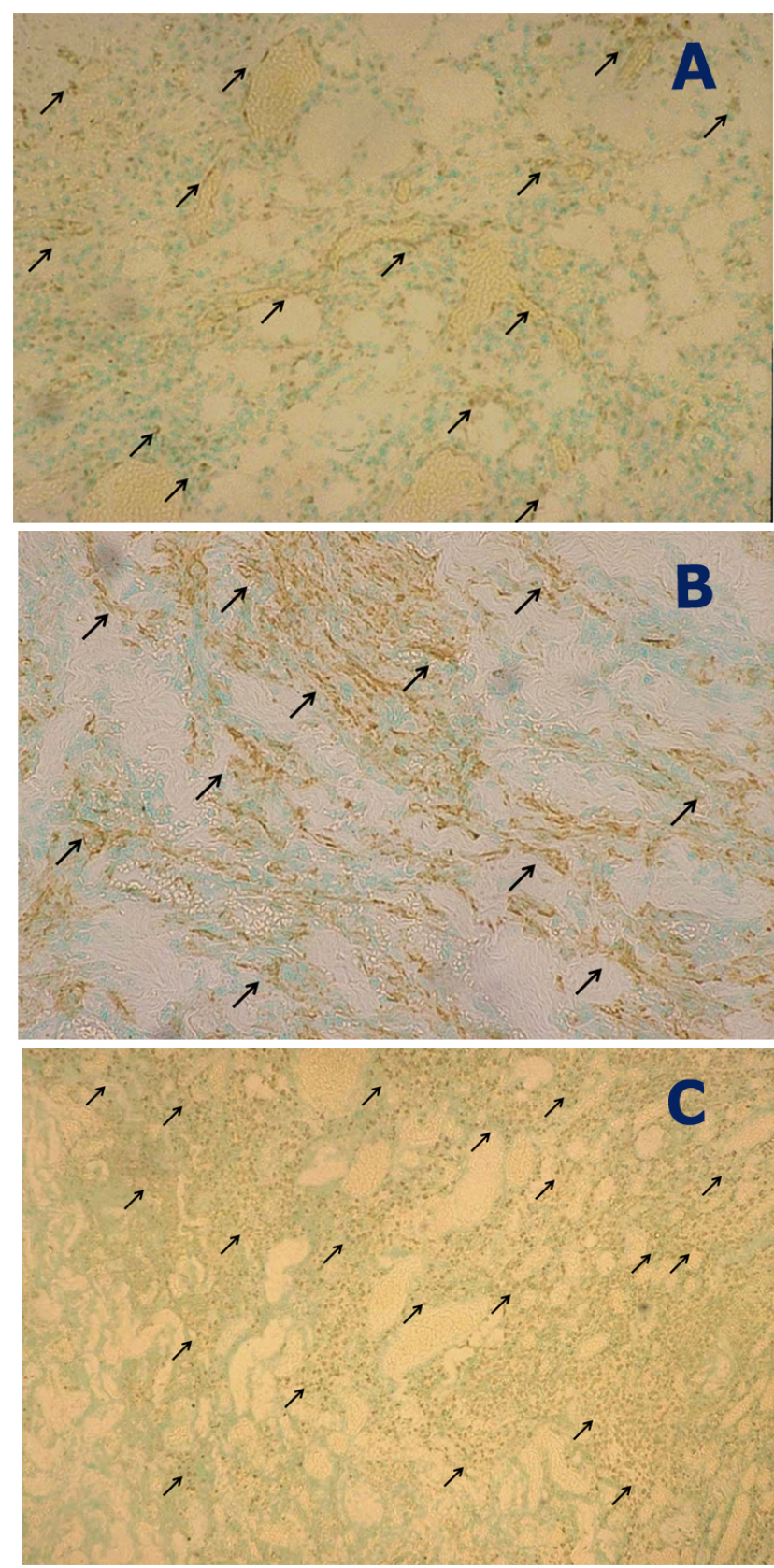

Figure 8. Immunohistochemical staining $(\times 50$, the color was developed by DAB)

A. Immunohistochemical staining for vimentin. Strongly positive in fibroblast cells around the inflammation region (arrows);

$B$. Immunohistochemical staining for $\alpha$-smooth muscle actin. Strongly positive around blood-corpuscle constituents (arrows);

C. Immunohistochemical staining for CD68(PGM1). Positive scattering at the inflammation region (arrows)

An inflammatory pseudotumor consists of proliferating spindle cells (fibroblast and myofibroblast). Its feature is infiltration of lymphocytes, plasma cells, histocytes and other inflammation cells. Hyalinization of stroma or nodular fasciitis are frequently observed. Inflammatory pseudotumors have been considered reactive lesions, but according to recent Published by Sciedu Press
DNA analysis and clinicopathologic findings, these lesions are generally considered tumors..$^{[1,2]}$

Many reports suggest that $\mathrm{CT}$ and MRI examination cannot distinguish between inflammatory pseudotumor and a benign or malignant tumor. We carried out immunohistological analyses and that showed that, fibroblasts adjacent to the inflammatory lesion were vimentin and $\alpha$-smooth muscle actin positivity in the stroma, but PCNA was not reactive. This lesion was composed of proliferations of spindled and rounded cells, which showed fascicular and storiform structures (see Figure 9). In the hyalinized connective tissue where the stroma was infiltrated, lymphocytes, neutrophils, plasma cells, and macrophage were extensive. Therefore, we concluded that this lesion was an inflammatory pseudotumor including clinical progress.

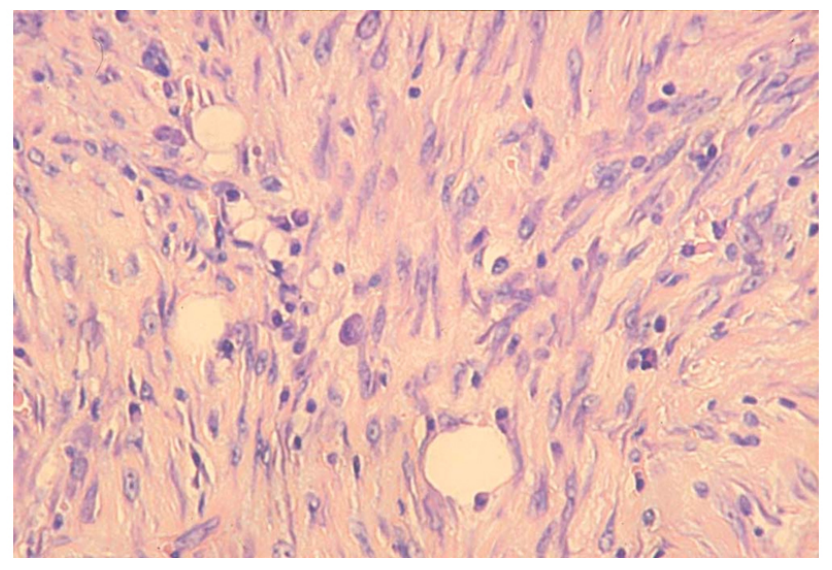

Figure 9. Fascicular and storiform structure (hematoxylin and eosin staining, $\times 100$ )

There are several reports of pseudotumors in the head and neck region, Liston (1981, buccal region), ${ }^{[3]}$ Earl (1993, mandibular angle region), ${ }^{[4]}$ Inui (1993, submandibular region), ${ }^{[5]}$ and Ide (1998, retromolar region). ${ }^{[6]}$ Ide reported immunohistochemically that the tumor was positive to vimentin, $\alpha$-smooth muscle, and CD68 (PGM1), and negative to $\mathrm{S}-100$ protein. Earl4 described a strong positive in the plasma of spindle cells, a variable expression of $\alpha$-smooth muscle, and negative for cytokeratin, desmin, and S-100 protein, which was similar to our results. In the majority of case reports of surgically treated inflammatory pseudotumors, the prognoses were comparatively good, but rare cases had recurred or transformed to a malignant condition (Coffin 1998). ${ }^{[1]}$ Therefore, we had observed this patient closely, and should be aware of the potential of aggressive character in inflammatory pseudotumor.

\section{CONFlicts OF INTEREST Disclosure}

The authors declare that they have no competing interests. 


\section{REFERENCES}

[1] Coffin CM, Watterson J, Priest JR, et al. Extrapulmonary Inflammatory Myofibroblastic Tumor (Inflammatory Pseudotumor). Am J Surg Pathol. 1995; 19(8): 895-872. http://dx.doi.org/10.1097/0 0000478-199508000-00001

[2] Biselli R, Ferlini C, Fattorossi A, et al. Inflammatory Myofibroblastic Tumor (Inflammatory Pseudotumor). Cancer. 1996; 77: 77884. http://dx.doi.org/10.1002/(SICI) 1097-0142(199602 15) $77: 4<778:$ :AID-CNCR25>3.0.CO ; $2-X$

[3] Liston SL, Dehner LP, Jarvis CW, et al. Inflammatory pseudotumor in the buccal tissues of children. Oral Surg Oral Med Oral Pathol. 1981; 51(3): 287-91. http://dx.doi.org/10.1016/0030-422 o(81) $90059-1$
[4] Earl PD, Lowry JC, Sloan P. Intraoral inflammatory pseudotumor. Oral Surg Oral Med Oral Pathol. 1993; 76: 279-83. http: //dx.doi.org/10.1016/0030-4220 (93) 90253-Z

[5] Inui M, Tagawa $\mathrm{T}$, Mori A, et al. Inflammatory pseudotumor in the submandibular region. Oral Surg Oral Med Oral Pathol. 1993; 76: 333-7. http://dx.doi.org/10.1016/0030-4220 (93) 902 63-4

[6] Ide F, Shimoyama T, Horie N. Inflammatory pseudotumor in the mandibular retromolar region. J Oral Pathol Med. 1998; 27: 508-10. PMid: 9831965. http://dx.doi.org/10.1111/j.1600-0714. 1998.tb01921. $x$ 